\title{
ESTADO NUTRICIONAL DE DIFERENTES CULTIVARES DE PEREIRA NAS CONDIÇÕES EDAFOCLIMÁTICAS DE GUARAPUAVA-PR ${ }^{1}$
}

\author{
RENATO VASCONCELOS BOTELHO², MARCELO MARQUES LOPES MÜLLER ${ }^{3}$, \\ $\mathrm{CLORI}^{\mathrm{BASSO}}{ }^{4}$, $\mathrm{ATSUO}^{\mathrm{S} U Z U K I}{ }^{5}$
}

RESUMO - A adubação de plantas frutíferas de clima temperado é rotineiramente recomendada em função da interpretação de laudos de análises químicas de solo e folhas, mas não são específicas para as diferentes cultivares e porta-enxertos. Neste contexto, um experimento foi conduzido durante dois anos, com o objetivo de avaliar o estado nutricional de diferentes cultivares de pereira enxertadas sobre o porta-enxerto marmeleiro 'CP', em Guarapuava-PR. As cultivares de pereira Cascatense, Tenra, Hosui, Packham `s Triumph e Williams foram plantadas em 2004, em densidade de 2.500 plantas ha $^{-1}$. Amostras de folhas e de frutos foram coletadas em 2006 e 2007 para análises químicas dos teores de nutrientes. Folhas completas e normais foram amostradas em meados de janeiro, retiradas da parte mediana das brotações do ano. Os frutos foram colhidos quando o teor de sólidos solúveis totais atingiram $10^{\circ}$ Brix. As cultivares de pereira apresentaram diferenças em relação aos teores de nutrientes nas folhas e frutos, demonstrando exigências nutricionais distintas. A cv. Cascatense apresentou os maiores teores de $\mathrm{N}$ e $\mathrm{P}$ nos frutos em, pelo menos, um dos anos avaliados, e a cv. Hosui, os maiores valores para K. A extração de nutrientes pelos frutos situou-se entre 0,366 e 0,825 $\mathrm{kg}$ de N; 0,097 e 0,205 kg de P; 0,996 e 1,302 kg de K; 0,085 e 0,049 a 0,085 kg de Ca, e entre 0,041 e $0,095 \mathrm{~kg}$ de $\mathrm{Mg}$ por tonelada de frutos.

Termos para indexação: Pyrus spp., Cydonia oblonga, análise de frutos, análise foliar, nutrição, portaenxerto.

\section{LEAF CONTENT AND NUTRIENT EXTRACTION BY FRUIT HARVEST OF PEAR TREES CULTIVARS GRAFTED ON QUINCE 'CP'}

\begin{abstract}
The fertilizer applications in deciduous fruit trees are usually recommended in function of chemical soil and leaves analysis interpretation, but they are not specific for the different scion and rootstock cultivars. In this context, a trial was carried out aiming to evaluate the nutritional status of different pear cultivars grafted on rootstock quince 'CP' by two consecutive years, in Guarapuava, state of Paraná, Brazil. The pear cultivars Cascatense, Tenra, Hosui, Packham's Triumph and Williams were planted in 2004 at the density planting of 2.500 plants ha $^{-1}$. Samples of leaves and fruits were collected in 2006 and 2007 for chemical analysis of nutrient contents. Complete and normal leaves were sampled in the end of January; taken from the middle part of one-year shoots. The fruits were collected when the total soluble solids content reached $10^{\circ}$ Brix. The pear cultivars showed differences in relation to nutrient content in leaves and fruits, indicating distinct nutritional requirements. The cv. Cascatense presented the highest $\mathrm{N}$ and $\mathrm{P}$ contents in fruits at least in one of the years, and the cv Hosui the highest $\mathrm{K}$ content. The nutrient extraction by fruits ranged from 0.366 to $0.825 \mathrm{~kg} \mathrm{~N} ; 0.097$ to $0.205 \mathrm{~kg} \mathrm{P}, 0.996$ to $1.302 \mathrm{~kg} \mathrm{~K}, 0.049$ to $0.085 \mathrm{~kg} \mathrm{Ca}$ and 0.041 to $0.095 \mathrm{~kg} \mathrm{Mg}$ per fruit ton.
\end{abstract}

Index terms: Pyrus spp. Cydonia oblonga, fruit analysis, leaf analysis, nutrition, rootstock.

\footnotetext{
1(Trabalho 144-09). Recebido em: 05-06-2009. Aceito para publicação em: 01-04-2010.

${ }^{2}$ Eng. Agr. Dr. Prof. Adjunto. Departamento de Agronomia, Universidade Estadual do Centro-Oeste - Unicentro. R. Simeão Varella de Sá no 03, CEP 85040-080-Guarapuava-PR. E-mail: rbotelho@unicentro.br

${ }^{3}$ Bolsista CNPq Pq-2.

${ }^{4}$ Eng. Agr. PhD Pesquisador em Fertilidade do Solo, EPAGRI/Estação Experimental de Caçador. C.P. 591, 89500-000 Caçador-SC. E-mail: clori@epagri.sc.gov.br

${ }_{5}^{5}$ Eng. Agr. MSc. Pesquisador em Fertilidade do Solo, EPAGRI/Estação Experimental de Caçador. C.P. 591, 89500-000 Caçador-SC. E-mail: suzuki@epagri.sc.gov.br
} 


\section{INTRODUÇÃO}

O Brasil produz cerca de $20.000 \mathrm{t}$ anuais de peras das espécies Pyrus communis L. e P. pyrina, em uma área de aproximadamente1.900 ha. Porém, consome cinco vezes mais do que produz, tendo importado 100 mil toneladas da fruta em 2005, equivalente a 50 milhões de dólares (MAPA, 2007; FAO, 2008). A expansão da cultura em território nacional tem encontrado entraves devido à baixa tecnologia de produção, como sistema de condução, poda, raleio, adubação, controle fitossanitário; indefinição e/ou inexistência de cultivares e porta-enxertos adaptados às diferentes condições edafoclimáticas e ainda à falta de mudas para atender aos fruticultores (NAKASU;LEITE, 1990).

De acordo com Wertheim et al. (2001), novos pomares estão sendo cultivados de maneira mais intensiva no mundo inteiro, em plantios mais adensados. As razões da mudança para sistemas de plantio em alta densidade são universais: retorno rápido de capital, economia de mão de obra, alta produtividade e qualidade de frutos. No entanto, a maioria dos porta-enxertos atualmente disponíveis para pereira confere excessivo vigor às copas e retarda a entrada em produção do pomar, não sendo adequados para plantios adensados (WEBSTER, 2001). Algumas seleções novas de porta-enxertos de marmeleiro (Cydonia oblonga) reduzem consideravelmente o vigor, induzem maior precocidade de produção, conferem boa produtividade do pomar e boa qualidade de frutos, mas ainda são moderadamente vigorosas, não podendo ser consideradas porta-enxertos anões. $\mathrm{O}$ marmeleiro $\mathrm{C}$ é o mais ananizante deles, Adams e Marmeleiro A são um pouco mais vigorosos e o BA-29 é classificado como semianão (WEBSTER, 2001; BERTELSEN; CALLESEN, 2001). No Brasil, ainda prevalecem os porta-enxertos Pyrus calleryana Decne, Pyrus betulaefolia Bunge e Pyrus communis L., que são bastante vigorosos e possuem sistema radicular abundante e vigoroso, o que confere à pereira a capacidade de explorar um grande volume de solo, buscando nutrientes também em camadas mais profundas (BASSO et al., 2003). Recentemente, foi lançado no Brasil o porta-enxerto marmeleiro 'CP' (Cydonia oblonga), desenvolvido pela Empresa Clone Propagação de Plantas (Araucária-PR), podendo ser classificado como um porta-enxerto anão, por reduzir consideravelmente o vigor das plantas e induzir precocidade, com florescimento e produção de frutos já no segundo ciclo vegetativo de condução das plantas (BOTELHO et al., 2005).

A adubação de plantas frutíferas de clima temperado é rotineiramente recomendada em função da interpretação de laudos de análises químicas de solo e folhas, mas não são específicas para as diferentes combinações de cultivares-copa e porta-enxertos. No entanto, as exigências nutricionais podem ser variáveis. De acordo com Stassen e North (2005), pereiras 'Forelle' apresentaram maiores teores de $\mathrm{N}$ e $\mathrm{P}$ nas folhas e de $\mathrm{K}, \mathrm{Ca}$ e $\mathrm{Mg}$ nas raízes, quando enxertadas sobre marmeleiro 'A', em comparação àquelas enxertadas sobre o híbrido de pereira 'BP1' (Pyrus sp.). Por outro lado, Singh et al. (2005) observaram grande variação na absorção de nutrientes entre 13 seleções de pereiras asiáticas enxertadas sobre o porta-enxerto 'Kainth'.

Para os Estados de SC e RS, para a adubação de manutenção da cultura da pereira, recomenda-se a análise conjunta dos seguintes parâmetros: análise foliar, análise do solo, idade das plantas, crescimento vegetativo, sistema de condução, adubações anteriores, produção, tratos culturais, distúrbios nutricionais e presença de sintomas de deficiência ou de toxidez (BASSO, 2003; CQFS-RS/SC, 2004).

$\mathrm{O}$ presente trabalho objetivou avaliar o teor de nutrientes em folhas e extração pelos frutos de diferentes cultivares de pereira enxertadas sobre o porta-enxerto marmeleiro 'CP' para subsidiar recomendações em programas de adubação e auxiliar no estabelecimento de parâmetros de avaliação do estado nutricional das plantas.

\section{MATERIAL E MÉTODOS}

O trabalho foi desenvolvido em pomar experimental da Fazenda-Escola da Universidade Estadual do Centro-Oeste, em Guarapuava-PR. As cultivares de pereira Cascatense, Tenra, Hosui, Packham's Triumph e Williams, enxertadas sobre o porta-enxerto anão marmeleiro ' $\mathrm{CP}$ ' (Cydonia oblonga), foram plantadas em 2004, no espaçamento de $1 \times 4 \mathrm{~m}$, conduzidas em sistema líder central, com copa estreita e com irrigação por gotejamento. As coordenadas geográficas locais são: $25^{\circ} 23^{\prime} 36^{\prime \prime}$ S $51^{\circ} 27^{\prime} 19^{\prime \prime} \mathrm{O}$; e $1.120 \mathrm{~m}$ de altitude. O clima é classificado como subtropical mesotérmico-úmido $(\mathrm{Cfb})$, sem estação seca, com verões frescos e invernos moderados (IAPAR, 2000). O solo da área experimental é um Latossolo Bruno distroférrico.

O delineamento experimental foi em blocos casualizados, com cinco tratamentos e cinco repetições de cinco plantas por parcela. Cada bloco com 25 plantas foi constituído por uma linha de plantio. Todas as práticas culturais, incluindo a adubação, foram realizadas de acordo com as 
recomendações técnicas para a cultura da pereira no Brasil (CENTELLAS-QUEZADA et al., 2003). No preparo do terreno, por ocasião do plantio em 2004, foram aplicados em área total os equivalentes a $2.500 \mathrm{~kg}$ de gesso agrícola, $1.250 \mathrm{~kg}$ de superfosfato simples, $420 \mathrm{~kg}$ de cloreto de potássio e $100 \mathrm{~kg}$ de MAP, por hectare, e 15 quilos de esterco de curral por metro linear de sulco de plantio. No primeiro ciclo, como adubação de crescimento, foram realizadas três aplicações de $20 \mathrm{~g}$ de sulfato de amônio por planta. No segundo ano, como adubação de manutenção, foram aplicadas $30 \mathrm{~g}$ de ureia, $30 \mathrm{~g}$ de superfosfato simples e $30 \mathrm{~g}$ de cloreto de potássio por planta, e no terceiro ano o dobro dessas quantidades. Em julho de 2005, ou seja, um ano após a implantação do pomar, foram retiradas amostras de solo a $0-20 \mathrm{~cm}$ e $20-40 \mathrm{~cm}$ na linha de plantio para análises químicas, cujos resultados são apresentados na Tabela 1.

Após a análise de solo, nos verões de 2006 e 2007 , respectivamente $2^{\circ}$ e $3^{\circ}$ ciclos depois da implantação do pomar, foram coletadas folhas para análise de macronutrientes (N, P, K, Ca e $\mathrm{Mg}$ ) e micronutrientes (Fe, Mn, Zn, Cu e B). Foram amostradas 40 folhas completas e normais por parcela experimental na segunda quinzena de janeiro, sendo retiradas da parte mediana das brotações do ano, as quais foram imersas em solução com detergente, enxaguadas com água destilada, secas a $65^{\circ} \mathrm{C}$ até peso constante, moídas e reservadas (BASSO et al., 2003). Para análise mineral da polpa, no ponto de colheita comercial de cada cultivar, ou seja, quando os frutos atingiram teor de sólidos solúveis totais de $10^{\circ} \mathrm{Brix}$, todas as peras foram colhidas de cada planta, sendo amostrados 10 frutos por parcela, selecionando-se aquelas normais e sem defeitos.

As análises químicas de solo e de folhas foram realizadas segundo metodologias descritas por Tedesco (1995). As análises químicas de fruto dos macronutrientes $\mathrm{N}, \mathrm{P}, \mathrm{K}, \mathrm{Ca}$ e $\mathrm{Mg}$ foram realizadas segundo metodologia descrita por Suzuki e Argenta (1994) para maçãs, na Estação Experimental da EPAGRI, em Caçador (SC). Para cada fruto, foi retirada uma fatia longitudinal de $1 \mathrm{~cm}$ de espessura, em forma de cunha, com casca, sem a parte central do carpelo. As amostras de polpa fresca foram trituradas em multiprocessador. Para a digestão, foi retirada uma amostra homogênea de aproximadamente 5,0 g para os tubos de digestão. A digestão foi feita utilizando-se de $\mathrm{H}_{2} \mathrm{O}_{2}+\mathrm{H}_{2} \mathrm{SO}_{4}$, segundo adaptação da metodologia descrita por Tedesco (1995), para tecido de planta. Os teores de nitrogênio foram determinados por Semi-Micro-Kjeldahl, os teores de K, Ca e Mg por espectrofotometria de absorção atômica, e os teores de $\mathrm{P}$, por determinação colorimétrica pelo método de vanádio-molibdato de amônio.

Os dados foram submetidos à análise de variância, e quando significativo, as médias foram comparadas pelo teste Tukey, ao nível de $5 \%$ de probabilidade.

\section{RESULTADOS E DISCUSSÃO}

As cultivares Packham`s Triumph, Williams e Cascatense apresentaram as maiores médias de $\mathrm{N}$ nas folhas (Tabela 2), nos dois anos de avaliação, estando ligeiramente acima do normal $\left(25,1\right.$ a $\left.30,0 \mathrm{~g} \mathrm{~kg}^{-1}\right)$, de acordo com recomendação de Basso et al. (2003) e da CQFS-RS/SC (2004) para a cultura da pereira no Brasil. As demais cultivares apresentaram teores de $\mathrm{N}$ dentro da faixa normal $\left(20,0\right.$ a $\left.25,0 \mathrm{~g} \mathrm{~kg}^{-1}\right)$. Em relação ao $P$, apenas as cultivares Packham 's Triumph e Williams apresentaram teores na faixa normal, nos dois anos, enquanto as demais cultivares tiveram teores de $\mathrm{P}$ abaixo do normal (1,00 a 1,40 $\left.\mathrm{g} \mathrm{kg}^{-1}\right)$ para a cultura, apresentando diferenças significativas. As cultivares Cascatense e Tenra apresentaram os menores teores de $\mathrm{K}$ nas folhas, estando abaixo do normal $\left(8,00\right.$ a $\left.11,90 \mathrm{~g} \mathrm{~kg}^{-1}\right)$ no ano de 2007, ou seja, 9,8 e 10,5 $\mathrm{g} \mathrm{kg}^{-1}$, respectivamente (Tabela 2). No entanto, nenhum dos teores de $\mathrm{Pe} \mathrm{K}$ se encontravam em níveis considerados insuficientes para a cultura de acordo com a recomendação de Basso et al. (2003) e da CQFS-RS/SC (2004), ou seja, $<1,0$ e $<8,0 \mathrm{~g} \mathrm{~kg}^{-1}$, respectivamente.

Os teores de $\mathrm{Ca}$ e $\mathrm{Mg}$ foram dentro da faixa normal para a cultura, nos dois anos de avaliação, ou seja, Ca entre 11,00 e 17,00 $\mathrm{g} \mathrm{kg}^{-1}$ e $\mathrm{Mg}$ entre 2,50 e 4,50 $\mathrm{g} \mathrm{kg}^{-1}$ (BASSO et al., 2003). Em 2006, a cv. Packham`s Triumph apresentou o maior teor de $\mathrm{Ca}$, diferindo-se apenas da cv. Cascatense, e a cv. Hosui, o maior teor de Mg. Em 2007, não houve diferenças significativas entre as cultivares para os teores de $\mathrm{Ca}$ e Mg (Tabela 2).

Os teores foliares mais elevados dos macronutrientes N, P e K nas cultivares Packham's Triumph e Williams, possivelmente ocorreram devido ao efeito concentração causado pelo menor desenvolvimento das partes aéreas das plantas (ramos, frutos, folhas). Segundo resultados de avaliações realizadas nesta mesma área experimental por Schreider et al. (2007), essas cultivares apresentaram brotação deficiente de, no máximo, 25\% de gemas em função da limitada adaptação ao clima da região de Guarapuava-PR, principalmente no que se refere às necessidades de frio para a quebra de dormência e à alta incidência de abortamento floral (SCHREIDER et al., 2007). De forma semelhante, Arzani et al. (2005) verificaram que, em plantas 
jovens de cultivares de pereira asiática com menor crescimento, também foram encontrados os maiores teores foliares de $\mathrm{N}, \mathrm{P}$ e K.

Os teores de $\mathrm{P}$ abaixo do normal, nas variedades com brotação uniforme, ou seja, Cascatense, Tenra e Hosui, podem estar relacionados aos teores de $\mathrm{P}$ do solo, médio na camada de $0-20 \mathrm{~cm}$ e baixo na camada de 20-40 cm (CQFS-RS/SC, 2004), o que se relaciona, em parte, ao $\mathrm{pH}$ em água do solo ligeiramente ácido, entre 5,8 e 4,9, principalmente na camada de 20 a $40 \mathrm{~cm}$ (Tabela 1), o que se relaciona à deficiência natural de $\mathrm{P}$ nos solos da região (EMBRAPA, 1984) e ao alto poder de adsorção de $\mathrm{P}$ em solos com alta proporção de argila (STILES, 1994), entre 500 e $630 \mathrm{~g} \mathrm{~kg}^{-1}$ no presente estudo.

Teores foliares de $\mathrm{K}$ abaixo do normal poderiam também estar relacionados, em parte, aos altos teores de $\mathrm{Ca}\left(>4,0 \mathrm{cmol}_{\mathrm{c}} \mathrm{dm}^{-3}\right)$ e $\mathrm{Mg}(>1,0$ $\mathrm{cmol}_{c} \mathrm{dm}^{-3}$ ) do solo (CQFS - RS/SC, 2004) que são antagonistas à absorção de K (STILES, 1994), interação conhecida como inibição competitiva, muito comum em plantas frutíferas. Como a ligação dos íons aos sítios de transporte da membrana plasmática é importante para a absorção em nível de célula, é lógico que haja competição entre os íons pelas cargas destes sítios (carregadores), e que cátions como $\mathrm{Ca}^{++}$ $\mathrm{e} \mathrm{K}^{+}$concorram efetivamente com $\mathrm{Mg}^{++}$(MARSCHNER, 1995). No entanto, o teor elevado de potássio de solo (160 $\left.\mathrm{mg} \mathrm{dm}^{-3}\right)$ torna essa influência de pouca importância. Dessa forma, possivelmente outras questões inerentes à natureza de cada interação copa/ porta-enxerto devem ter influenciado de modo mais marcante a nutrição mineral, pois suas exigências e capacidades de absorção são distintas.

Segundo Arzani et al. (2005), em estudo com nove cultivares de pereira asiática (Pyrus serotina) enxertadas sobre 'seedlings' de Pyrus communis, houve diferenças significativas para os teores foliares de N, P e K. De forma semelhante, Stassen e North (2005) verificaram que a pereira $\mathrm{cv}$. Forelle, quando enxertada sobre o porta-enxerto anão marmeleiro 'A' (Cydonia oblonga), apresentou menores teores foliares de $\mathrm{P}, \mathrm{Ca}$ e $\mathrm{Mg}$, e maiores de $\mathrm{N}$ e K, quando comparado ao porta-enxerto vigoroso 'BP1' (Pyrus communis).

Para os micronutrientes, a variabilidade foi muito grande para os teores foliares de $\mathrm{Fe}, \mathrm{Zn}$, $\mathrm{Mn}$ e $\mathrm{Cu}$, principalmente no primeiro ano, devido ao maior número de aplicações de fungicidas que contêm micronutrientes em sua formulação, o que contribuiu para que não fossem verificadas diferenças significativas entre os tratamentos em 2006. A maioria das cultivares apresentou teores foliares adequados de acordo com recomendação de Basso et al. (2003) e da CQFS-RS/SC (2004) para a cultura da pereira no Brasil, com exceção da cv. Hosui que, em 2006, apresentou valor significativamente mais baixo de B e, em 2007, valores mais baixos de $\mathrm{Zn}$, $\mathrm{Cu}$ e B (Tabela 3). Esses resultados podem indicar menor habilidade da cv. Hosui para absorção e transporte desses micronutrientes, ou até mesmo certa incompatibilidade com o porta-enxerto marmeleiro 'CP', o que deveria ser levado em consideração para recomendações de adubação. Singh et al. (2005) também verificaram diferenças significativas entre 13 cultivares de pereiras asiáticas, com teores variando para os seguintes micronutrientes Mn (112 a $135 \mathrm{mg}$ $\left.\mathrm{kg}^{-1}\right), \mathrm{Fe}\left(148\right.$ a $\left.209 \mathrm{mg} \mathrm{kg}^{-1}\right), \mathrm{Zn}\left(24\right.$ a $\left.50 \mathrm{mg} \mathrm{kg}^{-1}\right) \mathrm{e}$ $\mathrm{Cu}\left(9\right.$ a $\left.18 \mathrm{mg} \mathrm{kg}^{-1}\right)$

Segundo Basso et al. (2003), não se dispõe atualmente de informações que permitam recomendar a análise de frutos como parâmetro de avaliação do estado nutricional da pereira e do potencial de conservação das peras em câmara fria. Mielke (2004) estudou o efeito de sistemas de condução e de portaenxertos na nutrição da pereira 'd'Anjou', mas apenas analisou o teor de nutrientes na casca dos frutos, não considerando a polpa, impossibilitando qualquer estimativa de extração de nutrientes pela colheita. Para maçãs, inúmeros trabalhos mostram que a análise do teor de nutrientes na polpa é bastante útil para prognosticar a ocorrência de distúrbios fisiológicos, como o "bitter-pit" e o potencial de armazenagem a frio. Para maçãs, são considerados ideais os seguintes teores de macronutrientes na polpa: $360 \mathrm{mg} \mathrm{kg}^{-1} \mathrm{de}$ $\mathrm{N}, 60$ a $120 \mathrm{mg} \mathrm{kg}^{-1}$ de P, 950 a $1050 \mathrm{mg} \mathrm{kg}^{-1} \mathrm{de} \mathrm{K}, \geq$ $50 \mathrm{mg} \mathrm{kg}^{-1}$ de Ca e de 38 a $43 \mathrm{mg} \mathrm{kg}^{-1}$ de $\mathrm{Mg}$ (IUCHI et al., 2001; SUZUKI; BASSO, 2002; NAVA et al., 2006).

De maneira geral, pelos resultados apresentados na Tabela 4, verificou-se que os teores de macronutrientes na polpa de peras foram superiores aos relatados para maçãs, o que pode significar maior habilidade dessa frutífera em redistribuir o elemento para os frutos e maior demanda por nutrientes, fato que deve ser levando em consideração para recomendações de adubação. Para essas variáveis, houve diferenças significativas entre as cultivares nos dois anos de avaliação, sendo que a cv. Cascatense apresentou maior extração de $\mathrm{N}$ e $\mathrm{P}$ em, pelo menos, um dos anos analisados. Para K, a cv. Hosui destacou-se, diferindo significativamente da cv. Tenra, em 2007. Para os teores de $\mathrm{Ca}$ e $\mathrm{Mg}$, as cultivares Packham`s Triumph e William's tiveram, de maneira geral, maiores valores, diferindo das demais cultivares em, pelo menos, um dos anos avaliados, possivelmente pela menor competição entre drenos, tendo em vista a baixa brotação das gemas e a baixa produtividade. 
Tendo em vista que atualmente não há recomendações oficiais de quantidades de nutrientes para aplicação na cultura da pereira para adubação de manutenção, na fase produtiva do pomar (BASSO et al., 2003; CQFS-RS/SC, 2004), torna-se relevante a indicação de extração de nutrientes pelos frutos. As cultivares Tenra, Cascatense e Hosui, enxertadas sobre o marmeleiro 'CP', apresentaram maior adaptação e brotação uniforme, não apresentaram teores foliares de nutrientes considerados insuficientes e/ ou sintomas de deficiência nutricional e produziram cumulativamente no $2^{\circ}$ e $3^{\circ}$ anos do pomar 13,$16 ; 6,49$ e 4,45 $\mathrm{t} \mathrm{ha}^{-1}$, respectivamente (SCHREIDER, 2007). Considerando os dados de produtividade e teores de nutrientes na polpa, é possível estimar as seguintes quantidades de nutrientes extraídas pela colheita nos dois primeiros anos de produção por hectare $\left(2^{\circ}\right.$ e $3^{\circ}$ anos após implantação): Tenra (3,974 kg de N, 2,138 $\mathrm{kg}$ de P, 13,708 $\mathrm{kg}$ de $\mathrm{K}, 0,628 \mathrm{~kg}$ de Ca, 0,650 kg de $\mathrm{Mg})$, Cascatense $(3,659 \mathrm{~kg}$ de N, 1,242 $\mathrm{kg}$ de $\mathrm{P}$, $7,110 \mathrm{~kg}$ de $\mathrm{K}, 0,350 \mathrm{~kg}$ de $\mathrm{Ca}, 0,426 \mathrm{~kg}$ de $\mathrm{Mg}$ ), Hosui $(1,864 \mathrm{~kg}$ de N, 0,779 $\mathrm{kg}$ de P, 5,767 kg de K, $0,232 \mathrm{~kg}$ de $\mathrm{Ca}, 0,256 \mathrm{~kg}$ de $\mathrm{Mg}$ ). Segundo Stassen e North (2005), para a pereira cv. Forelle sobre o portaexerto anão marmeleiro 'A' (Cydonia oblonga), nas condições da África do Sul, o mínimo requerimento de nutriente por tonelada de frutos produzidos é de $2,9 \mathrm{~kg}$ de N, 0,470 $\mathrm{kg}$ de $\mathrm{P}, 1,83 \mathrm{~kg}$ de $\mathrm{K}, 1,75 \mathrm{~kg}$ de $\mathrm{Ca}$ e $0,49 \mathrm{~kg}$ de $\mathrm{Mg}$, sendo que desses totais são exportados pelos frutos $27,2 \%$ de N, 39,3\% de P, $50,3 \%$ de $\mathrm{K}, 1,6 \%$ de $\mathrm{Ca}$ e $14,2 \%$ de $\mathrm{Mg}$. As demais quantidades dos nutrientes são perdidas na poda, na queda das folhas ou são fixadas pelas partes permanentes da planta, sendo que, quando os materiais de poda e as folhas permanecem no pomar, entram no ciclo da matéria orgânica do solo e, portanto, há reciclagem dos nutrientes.

TABELA 1 - Características físicas e químicas das amostras de solo nas profundidades de 0-20 cm e 20-40 $\mathrm{cm}$ de pomar experimental com diferentes cultivares de pereira (Guarapuava, 2005).

\begin{tabular}{lcc}
\hline \multicolumn{1}{c}{ Atributo $^{(\mathbf{1})}$} & $\mathbf{0 - 2 0} \mathbf{~ c m}$ & $\mathbf{2 0 - 4 0} \mathbf{~ c m}$ \\
\hline Argila, $\mathrm{g} \mathrm{kg}^{-1}$ & 500 & 630 \\
Matéria orgânica, $\mathrm{g} \mathrm{kg}^{-1}$ & 4,2 & 3,1 \\
$\mathrm{pH}-\mathrm{H}_{2} 0$ & 5,8 & 5,3 \\
Índice SMP & 6,1 & 6,1 \\
$\mathrm{Al}$ trocável, $\mathrm{cmol}_{\mathrm{c}} \mathrm{dm}^{-3}$ & 0,0 & 0,2 \\
Ca trocável, $\mathrm{cmol}_{\mathrm{c}} \mathrm{dm}^{-3}$ & 6,4 & 3,5 \\
P disponível, $\mathrm{mg} \mathrm{dm}^{-3}$ & 5,3 & 3,3 \\
K disponível, mg dm$^{-3}$ & 160 & 62 \\
Saturação de bases (\%) & 70,97 & 60,50 \\
\hline
\end{tabular}

(1) Metodologia analítica conforme Tedesco (1995). 
TABELA 2 -Teores de nitrogênio, fósforo, potássio, cálcio e magnésio $\left(\mathrm{g} \mathrm{kg}^{-1}\right)$ em folhas de pereiras enxertadas sobre o marmeleiro ' $\mathrm{CP}$ ', no $2^{\circ}$ e $3^{\circ}$ anos do pomar, em Guarapuava-PR, nas safras de 2006 e 2007.

\begin{tabular}{|c|c|c|c|c|c|}
\hline Nutrientes & Nitrogênio & Fósforo & Potássio & Cálcio & Magnésio \\
\hline Teor Normal & $20-25^{1}$ & $1,5-3,0$ & $12,0-15,0$ & $11,0-17,0$ & $2,5-5,0$ \\
\hline & \multicolumn{5}{|c|}{2006} \\
\hline \multicolumn{6}{|l|}{ Cultivares } \\
\hline Cascatense & $25,6 a^{2}$ & $1,43 \mathrm{~b}$ & $12,4 \mathrm{~b}$ & $12,6 \mathrm{~b}$ & $4,20 \mathrm{~b}$ \\
\hline Tenra & $24,1 \mathrm{~b}$ & $1,40 \mathrm{~b}$ & $12,9 \mathrm{~b}$ & $15,9 \mathrm{ab}$ & $3,97 \mathrm{~b}$ \\
\hline Hosui & $26,1 \mathrm{ab}$ & $1,47 \mathrm{ab}$ & $16,9 \mathrm{a}$ & $16,3 \mathrm{ab}$ & $5,53 \mathrm{a}$ \\
\hline Packham's & $27,6 \mathrm{a}$ & $1,67 \mathrm{ab}$ & $14,7 \mathrm{ab}$ & $17,1 \mathrm{a}$ & $4,27 \mathrm{~b}$ \\
\hline Williams & $28,5 \mathrm{a}$ & $1,80 \mathrm{a}$ & $13,4 \mathrm{ab}$ & $16,0 \mathrm{ab}$ & $4,37 \mathrm{~b}$ \\
\hline $\operatorname{Pr}>\mathrm{F}$ & 0,0132 & 0,014 & 0,016 & 0,024 & 0,0089 \\
\hline DMS & 3,31 & 0,33 & 3,63 & 3,79 & 1,11 \\
\hline CV (\%) & 4,45 & 7,71 & 9,16 & 8,64 & 8,82 \\
\hline \multicolumn{6}{|c|}{2007} \\
\hline \multicolumn{6}{|l|}{ Cultivares } \\
\hline Cascatense & $24,1 \mathrm{abc}^{2}$ & $1,27 \quad \mathrm{~cd}$ & $9,8 \mathrm{~b}$ & $12,7 \mathrm{a}$ & $4,50 \mathrm{a}$ \\
\hline Tenra & 20,9 bc & $1,30 \mathrm{bc}$ & $10,5 \mathrm{ab}$ & $16,5 \mathrm{a}$ & $4,13 a$ \\
\hline Hosui & $20,3 \quad c$ & $1,07 \quad d$ & $12,5 \mathrm{ab}$ & $13,8 \mathrm{a}$ & $4,67 \mathrm{a}$ \\
\hline Packham's & $25,6 \mathrm{a}$ & $1,53 \mathrm{a}$ & $13,4 a$ & $14,4 \mathrm{a}$ & $3,47 \mathrm{a}$ \\
\hline Williams & $27,4 a$ & $1,50 \mathrm{ab}$ & $11,6 a b$ & $13,5 \mathrm{a}$ & $3,83 \mathrm{a}$ \\
\hline $\operatorname{Pr}>F$ & 0,0007 & 0,0005 & 0,019 & 0,3933 & 0,2138 \\
\hline DMS & 3,76 & 0,22 & 3,03 & 6,49 & 1,75 \\
\hline CV $(\%)$ & 5,64 & 5,97 & 9,34 & 16,24 & 15,11 \\
\hline
\end{tabular}

${ }^{1}$ Teores foliares considerados normais para a cultura da pereira para os Estados do Rio Grande do Sul e Santa Catarina (CQFS-RS/SC, 2004). ${ }^{2}$ Médias seguidas por letras iguais na mesma coluna não diferem entre si, pelo teste Tukey, ao nível de $5 \%$ de probabilidade.

TABELA 3 - Teores de ferro, manganês, zinco, cobre e boro $\left(\mathrm{mg} \mathrm{kg}^{-1}\right)$ em folhas de pereiras enxertadas sobre o marmeleiro 'CP', no $2^{\circ}$ e $3^{\circ}$ anos do pomar, em Guarapuava-PR, nas safras de 2006 e 2007.

\begin{tabular}{|c|c|c|c|c|c|}
\hline Nutrientes & Ferro & Manganês & $\begin{array}{l}\text { Zinco } \\
\end{array}$ & Cobre & Boro \\
\hline Teor Normal & $50-250^{1}$ & $30-130$ & $20-100$ & 5 a 30 & 25 a 50 \\
\hline & & & 2006 & & \\
\hline \multicolumn{6}{|l|}{ Cultivares } \\
\hline Cascatense & 124,7 & 347,7 & 67,7 & 15,3 & $28,7 \mathrm{a}^{2}$ \\
\hline Tenra & 127,7 & 117,7 & 29,3 & 44,7 & $22,0 \mathrm{bc}$ \\
\hline Hosui & 141,3 & 90,7 & 25,0 & 14,3 & $21,0 \quad \mathrm{c}$ \\
\hline Packham's & 89,3 & 129,7 & 32,0 & 18,3 & $25,7 \mathrm{abc}$ \\
\hline Williams & 104,3 & 114,3 & 34,0 & 20,3 & $27,7 \mathrm{ab}$ \\
\hline $\operatorname{Pr}>\mathrm{F}$ & 0,0774 & 0,045 & 0,055 & 0,243 & 0,0079 \\
\hline DMS & 56,42 & 259,35 & 43,73 & 47,19 & 6,01 \\
\hline \multirow[t]{2}{*}{ CV $(\%)$} & 17,03 & 57,45 & 41,22 & 74,0 & 8,53 \\
\hline & & & 2007 & & \\
\hline \multicolumn{6}{|l|}{ Cultivares } \\
\hline Cascatense & 90,3 & 92,0 & $28,3 a$ & $10,0 \mathrm{a}$ & $19,3 \mathrm{a}$ \\
\hline Tenra & 76,7 & 81,0 & $25,3 \mathrm{ab}$ & $8,0 \mathrm{a}$ & $17,0 \mathrm{ab}$ \\
\hline Hosui & 86,3 & 81,7 & $21,7 \mathrm{~b}$ & $5,3 \mathrm{~b}$ & $12,7 \mathrm{~b}$ \\
\hline Packham's & 87,7 & 98,0 & $26,7 \mathrm{a}$ & $10,0 \mathrm{a}$ & $21,0 \mathrm{a}$ \\
\hline Williams & 88,3 & 93,0 & $28,7 \mathrm{a}$ & $9,0 \mathrm{a}$ & $20,7 \mathrm{a}$ \\
\hline $\operatorname{Pr}>\mathrm{F}$ & 0,2203 & 0,5188 & 0,0048 & 0,0012 & 0,0082 \\
\hline DMS & 19,40 & 39,04 & 4,63 & 2,54 & 6,13 \\
\hline CV $(\%)$ & 8,01 & 15,53 & 6,29 & 10,67 & 12,00 \\
\hline
\end{tabular}

${ }^{1}$ Teores foliares considerados normais para a cultura da pereira para os Estados do Rio Grande do Sul e Santa Catarina (CQFS-RS/ SC, 2004), ${ }^{2}$ Médias seguidas pela mesma letra na coluna não diferem entre si, pelo teste Tukey, ao nível de $5 \%$ de probabilidade. 
TABELA 4 - Teores de nitrogênio, fósforo, potássio, cálcio e magnésio ( $\mathrm{mg} \mathrm{kg}^{-1}$ polpa fresca) em frutos de diferentes cultivares de pereira, no $2^{\circ}$ e $3^{\circ}$ anos do pomar, em Guarapuava-PR, nas safras de 2006 e 2007.

\begin{tabular}{lccccc}
\hline Nutrientes & $\mathbf{N}$ & $\mathbf{P}$ & $\mathbf{K}$ & $\mathbf{C a}$ & $\mathbf{M g}$ \\
\hline \multicolumn{7}{c}{ Cultivares } & & 2006 & & \\
Cascatense & $825,3 \mathrm{a}^{1}$ & $126,0 \mathrm{a}$ & 1258,3 & $76,66 \mathrm{ab}$ & $89,3 \mathrm{a}$ \\
Tenra & $588,0 \mathrm{~b}$ & $97,7 \mathrm{~b}$ & 1145,7 & $57,33 \mathrm{~b}$ & $70,0 \mathrm{~b}$ \\
Hosui & $612,0 \mathrm{~b}$ & $100,0 \mathrm{ab}$ & 1302,3 & $61,00 \mathrm{~b}$ & $90,7 \mathrm{a}$ \\
Packham's & $639,0 \mathrm{ab}$ & $121,0 \mathrm{ab}$ & 1136,7 & $64,00 \mathrm{~b}$ & $94,7 \mathrm{a}$ \\
William's & $575,0 \mathrm{~b}$ & $121,0 \mathrm{ab}$ & 1141,0 & $85,00 \mathrm{a}$ & $102,0 \mathrm{a}$ \\
\hline Pr $>$ F & 0,013 & 0,020 & 0,025 & 0,011 & 0,004 \\
D.M.S. & 199,11 & 28,30 & 172,79 & 20,37 & 17,91 \\
CV (\%) & 9,95 & 8,21 & 4,70 & 9,83 & 6,61 \\
\hline \multicolumn{7}{c}{2007} \\
Cultivares & & & & \\
Cascatense & $545,0 \mathrm{a} 2$ & 205,0 & $1083,6 \mathrm{ab}$ & $52,4 \mathrm{bc}$ & $58,7 \mathrm{~b}$ \\
Tenra & $422,4 \mathrm{~b}$ & 188,0 & $996,6 \mathrm{~b}$ & $43,8 \mathrm{c}$ & $41,0 \mathrm{c}$ \\
Hosui & $366,6 \mathrm{~b}$ & 195,8 & $1292,8 \mathrm{a}$ & $49,8 \mathrm{bc}$ & $48,7 \mathrm{c}$ \\
Packham's & $452,7 \mathrm{~b}$ & 194,7 & $1189,3 \mathrm{ab}$ & $91,3 \mathrm{a}$ & $77,0 \mathrm{a}$ \\
William's & $460,5 \mathrm{ab}$ & 208,5 & $1102,5 \mathrm{ab}$ & $69,0 \mathrm{~b}$ & $77,3 \mathrm{a}$ \\
\hline Pr $>$ F & 0,0001 & 0,235 & 0,020 & 0,0002 & 0,0001 \\
D.M.S. & 90,77 & 31,21 & 282,62 & 21,97 & 9,34 \\
CV (\%) & 8,10 & 6,46 & 10,19 & 15,71 & 5,46 \\
\hline
\end{tabular}

${ }^{1}$ Médias seguidas por letras iguais numa mesma coluna não diferem entre si, pelo teste Tukey, ao nível de 5\% de probabilidade.

\section{CONCLUSÕES}

1-As diferentes cultivares de pereira enxertadas sobre o porta-enxerto ' $\mathrm{CP}$ ' apresentam diferenças em relação aos teores de nutrientes nas folhas e frutos, demonstrando exigências nutricionais distintas.

2-A cv. Hosui, de origem asiática, apresentou teores foliares de $\mathrm{Cu}, \mathrm{Zn}$ e $\mathrm{B}$ mais baixos, e maiores valores de $\mathrm{K}$ nos frutos, sugerindo requerimentos diferenciados em relação às outras cultivares de origem europeia ou híbridas, ou mesmo, algum grau de incompatibilidade com o porta-enxerto ' $\mathrm{CP}$ '.

3-A extração de nutrientes pelos frutos situase entre 0,366 e $0,825 \mathrm{~kg}$ de N; 0,097 e 0,205 kg de P; 0,996 e 1,302 kg de K; 0,049 e 0,085 kg de Ca, e entre 0,041 e $0,095 \mathrm{~kg}$ de $\mathrm{Mg}$ por tonelada de frutos.

\section{AGRADECIMENTOS}

Ao Conselho Nacional de Desenvolvimento Científico e Tecnológico - CNPq, e à Fundação Araucária de Apoio ao Desenvolvimento Científico e Tecnológico do Paraná, pela concessão de auxílios financeiros e bolsas para condução desta pesquisa.

\section{REFERÊNCIAS}

ARZANI, K.; KHOSHGHALB, H.; KARIMZA$\mathrm{DEH}, \mathrm{G}$. Scion/rootstock influence on grafting success, early performance, tree survival and efficiency of nutrient uptake of some asian pear (Pyrus serotina Rehd.) cultivars. Acta Horticulturae, Wageningen, n.671, p. 477-480, 2005.

BERTELSEN, M.; CALLESSEN, O. Performance of 'Clara Frijs' pear on seven rootstocks. Acta Horticulturae, Wageningen, n.557, p.145-150, 2001. 
BOTELHO, R. V.; MULLER, M. M. L.; POTT, C. A.; DENEGA, S. Formação de pomaresa da pereira asiática cv. Hosui (Pyrus pyrifolia) em sistemas de alta e média densidades no terceiro planalto paranaense. In: SEMANA DE PESQUISA DA UNICENTRO, 17., 2005, Guarapuava. Anais... Guarapuava: Editora Unicentro, 2005. CD-ROM

BASSO, C.; FREIRE, C.J.S.; SUZUKI, A. Solos, adubação e nutrição. In: CENTELLAS-QUEZADA, A.C.; NAKASU, B.H.; HERTER, F.G. (Ed.). Pera: produção. Brasília: Embrapa, 2003. p. 55-67. (Frutas do Brasil, 46).

CENTELLAS-QUEZADA, A.; NAKASU, B.H.; HERTER, F.G. (Ed.). Pera: produção. Brasília: Embrapa, 2003. 105p. (Frutas do Brasil, 46)

CQFS-COMISSÃO DE QUÍMICA E FERTILIDADE DO SOLO-RS/SC. Manual de adubação e de calagem para os Estados do Rio grande do Sul e de Santa Catarina. 10. ed. Porto Alegre: SBCS-Núcleo Regional Sul, 2004. 400p

FAO - Food and Agriculture Organization of the United Nations. FAO Statistic Division, 2008. Disponível em: <www.faostat.fao.org > Acesso em: 16 jun. 2008.

IAPAR - Instituto Agronômico do Paraná. Cartas climáticas do Paraná. Versão 1.0. Londrina: IAPAR, 2000. CD-ROM

IUCHI, V.L.; NAVA, G.; IUCHI, T. Distúrbios fisiológicos e desequilíbrios nutricionais em macieiras. Florianópolis: EPAGRI, 2001. 74p.

MAPA - Ministério da Agricultura, Pecuária e Abastecimento. Agricultura brasileira em números. Disponível em <http//:www.agricultura.gov.br>. Acesso em: 15 jun. 2007.

MARSCHNER, H. Mineral nutrition of higher plants. $2^{\text {nd }}$ ed. London: Academic Press, 1995. 889 p.

MIELKE, E.A. Effects of rootstock and training system on fruit quality and peel nutrient content in 'd'Anjou pears: II. Peel nutrient content. Journal of Tree Fruit Production, Chicago, v.3, n.2, p.5774, 2004.

NAKASU, B.H.; LEITE, D.L. Indicação de portaenxertos e cultivares de pereira para o sul do Brasil. Hortisul, Nova Petrópolis, v.1, n.2, p.20-24, 1990.
NAVA, G.; BASSO, C.; NACHTIGALL, G.R.; MELO, G.W.B. de; SUZUKI, A. Conceitos e práticas sobre fertilidade do solo e nutrição na produção integrada de maçã. In: SANHUEZA, R.M.V.; PROTAS, J.S.F.; FREIRE, J.M. Manejo da macieira no sistema de produção integrada de frutas. Bento Gonçalves: Embrapa Uva e Vinho, 2006. p.81-91.

SCHREIDER, E.; BOTELHO, R. V.; PIVA, R. Produtividade e desevolvimento de pereiras (Pyrus spp) em plantio adensado em Guarapuava-PR. In: ENCONTRO ANUAL DE INICIAÇÃO CIENTÍFICA, 16., 2007, Florianópolis. Anais... Maringá: Universidade Estadual de Maringá, 2007.

SINGH, T.; SANDHU, A.S.; SINGH, R.; DHILLON W.S. Vegetative and fruiting behavior of semi-soft pear strains in relation to nutrient status. Acta Horticulturae, Wageningen, n.696, p.289-293, 2005.

STASSEN, P.J.C.; NORTH, M.S. Nutrient distribution and requirement of 'Forelle' pear trees on two rootstocks. Acta Horticulturae, Wageningen, n.671, p.493-500, 2005.

STILES, W. Phosphorus, potassium, magnesium, and sulfur soil management. In: PETERSON, A.B.; STEVENS, R.G. Tree fruit nutrition. Washington: Good Fruit Grower, 1994. p.31-40.

SUZUKI, A.; ARGENTA, L.C. Teores minerais na polpa das cvs. Gala, Golden Delicious e Fuji. Revista Brasileira de Fruticultura, Jaboticabal, v.16, n.1, p.92-104, 1994.

SUZUKI, A.; BASSO, C. Solos e nutrição da macieira. In: EPAGRI. A cultura da macieira. Florianópolis, 2002. p.341-380.

TEDESCO, M.J.; GIANELLO, C.; BISSANI, C.A.; BOHNEN, H.; VOLKWEISS, S.J. Análise do solo, planta e outros materiais. 2.ed. Porto Alegre: UFRGS, Departamento de Solos, 1995. 174p. (Boletim Técnico, 5).

WEBSTER, A.D. Rootstocks for temperate fruit crops: current uses, future potential and alternative strategies. Acta Horticultae, Wageningen, n.557, p.25-34, 2001.

WERTHEIM, S.J.; WAGENMAKERS, P.S.; BOOTSMA, J.H.; GROOT, M.J. Orchard systems for apple and pear: conditions for success. Acta Horticulturae, Leiden, n.557, p.209-227, 2001. 